\title{
Consonance and Cantor set-selectors
}

Valentin Gutev ${ }^{1 *}$

1 Department of Mathematics, Faculty of Science, University of Malta, Msida, MSD 2080, Malta

Received 1 December 2011; accepted 19 April 2012

Abstract: It is shown that every metrizable consonant space is a Cantor set-selector. Some applications are derived from this fact, also the relationship is discussed in the framework of hyperspaces and Prohorov spaces.

MSC:

$54 \mathrm{C} 60,54 \mathrm{C} 65,54 \mathrm{~B} 20$

Keywords: Set-valued mapping $\bullet$ Lower (upper) semi-continuous $\bullet$ Selection $\cdot$ Section $•$ Consonant space

(C) Versita Sp. z o.o.

\section{Introduction}

For a space $X$, let $\mathcal{F}(X)$ be the collection of all nonempty closed subsets of $X$, and $\mathcal{C}(X)$ - that of all compact members of $\mathcal{F}(X)$. An interesting class of spaces was introduced by Dolecki, Greco and Lechicki in [11, 12]: a space $X$ was called consonant if on the hyperspace $\mathcal{F}(X)$ the co-compact topology coincides with the upper Kuratowski topology. This class became of great interest especially after it was noticed by Nogura and Shakhmatov [25] that two other classical hyperspace topologies, the Fell and Kuratowski topologies, (automatically) coincide on $\mathcal{F}(X)$ provided $X$ is consonant.

It was proved in [12] that Čech-complete spaces are consonant. It is also known that metrizable consonant spaces are hereditarily Baire [4]. These results prompted Nogura and Shakhmatov to ask whether consonant metrizable spaces are Čech-complete [25, Problem 11.4]. The question was resolved by Bouziad [6] by showing that the statement all analytic metrizable consonant spaces are completely metrizable is independent of usual axioms for set theory.

Consonant spaces can be described in terms out of the framework of hyperspace topologies. One way (following the original definition in $[11,12])$ is for a space $X$ to consider the lattice $\mathcal{T}$ of all open subsets of $X$ equipped with the Scott topology, see [29]. A Scott open subset $\mathcal{H} \subset \mathcal{T}$, called also a compact family, is such that $U \in \mathcal{H}$ for every $U \in \mathcal{T}$ which contains an element of $\mathcal{H}$; and if $\bigcup \mathcal{U} \in \mathcal{H}$ for some $\mathcal{U} \subset \mathcal{T}$, then $\bigcup \mathcal{V} \in \mathcal{H}$ for some finite $\mathcal{V} \subset \mathcal{U}$. Now, a space $X$ is consonant if and only if each Scott open set $\mathcal{H} \subset \mathcal{T}$ is compactly generated, i.e. for each $U \in \mathcal{H}$ there exists a compact

\footnotetext{
*E-mail:vggutev@gmail.com
} 
subset $K \subset X$ such that $V \in \mathcal{H}$ for every $V \in \mathcal{T}$, with $K \subset V$. Another more geometrical way is in terms of sections for set-valued mappings, it was suggested by Bouziad in [6], see also [7]. In order to state it, recall that a set-valued mapping $\varphi: Z \rightarrow \mathcal{F}(X)$ is lower semi-continuous (l.s.c.) if the set $\varphi^{-1}[U]=\{z \in Z: \varphi(z) \cap U \neq \varnothing\}$ is open in $Z$ for every open $U \subset X$. Following [6], we say that a subset $A \subset X$ is a section for $\varphi: Z \rightarrow \mathcal{F}(X)$ if $A \cap \varphi(z) \neq \varnothing$ for every $z \in Z$. In particular, we say that a section $A$ for $\varphi$ is open (compact, etc.) if $A$ is an open (respectively, compact, etc.) subset of $X$.

Theorem 1.1 ([6]).

A space $X$ is consonant if and only if for every l.s.c. mapping $\varphi: Z \rightarrow \mathcal{F}(X)$, where $Z$ is a compact space, every open section $U \subset X$ for $\varphi$ contains a compact section $K \subset X$ for $\varphi$.

In this characterisation, compact spaces are not assumed to be Hausdorff, consequently compact subsets of these spaces are not necessarily closed.

A mapping $\theta: Z \rightarrow \mathcal{F}(X)$ is upper semi-continuous (u.s.c.) if the set $\varphi^{-1}[F]$ is closed in $Z$ for every closed $F \subset X$; and $\theta$ is called usco it if is nonempty-compact-valued and u.s.c. Let $A \subset X$ be a compact section for $\varphi: Z \rightarrow \mathcal{F}(X)$. Then, one can define a usco mapping $\theta: Z \rightarrow \mathcal{C}(X)$ by $\theta(z)=A, z \in Z$, which now has the property that $\theta(z) \cap \varphi(z) \neq \varnothing$ for every $z \in Z$. Such mappings were called sections in [15], i.e. the above $\theta$ is a usco section for $\varphi$. Finally, a mapping $\theta: Z \rightarrow \mathcal{F}(X)$ is a multi-selection (or, a set-valued selection) for $\varphi: Z \rightarrow \mathcal{F}(X)$ if $\theta(z) \subset \varphi(z)$ for every $z \in Z$.

If $X$ is a completely metrizable space and $Z$ is paracompact, then every l.s.c. mapping $\varphi: Z \rightarrow \mathcal{F}(X)$ has a usco multi-selection $\theta: Z \rightarrow \mathcal{C}(X)[22$, Theorem 1.1]. Conversely, if $X$ is a metrizable space with the property that for every paracompact space $Z$, every l.s.c. mapping $\varphi: Z \rightarrow \mathcal{F}(X)$ has a usco multi-selection $\theta: Z \rightarrow \mathcal{C}(X)$, then $X$ must be Čech-complete (i.e., completely metrizable) [24, Proposition 6], see also [28, Lemma 2]. Motivated by this, a space $X$ was called a Cantor set-selector, or a $\mathfrak{C}$-selector [16], provided every l.s.c. mapping $\varphi: \mathfrak{C} \rightarrow \mathcal{F}(X)$, from the Cantor set $\mathfrak{C}$, has a u.s.c. multi-selection $\theta: \mathfrak{C} \rightarrow \mathcal{F}(X)$. In [16] there was considered a question whether every metrizable $\mathfrak{C}$-selector is Čech-complete (equivalently, completely metrizable). The question was resolved by van Mill, Pelant and Pol [23] by showing that the statement all analytic metrizable $\mathfrak{C}$-selectors are completely metrizable is independent of usual axioms for set theory. This motivated the author to pose the following question in [15].

Question 1 ([15]).

Is every metrizable consonant space a $\mathfrak{C}$-selector?

In this paper we prove the following theorem which provides the solution to Question 1.

Theorem 1.2.

Every metrizable consonant space is a $\mathfrak{C}$-selector.

Theorem 1.2 has a remarkably simple proof, it is presented in the next section. This proof is based on the fact that every $G_{\delta}$-subset of a metrizable consonant space is consonant as well, see Section 2 . This fact is related to properties of hyperspaces over consonant spaces. They are discussed in Section 3, where we show that "all" Vietoris hyperspaces of compact subsets of metrizable consonant spaces are consonant, see Theorem 3.2 and Corollary 3.3. This reveals a stronger property of metrizable consonant spaces that all such hyperspaces are also $\mathfrak{C}$-selectors. Another ingredient implicitly involved in the solution of Question 1 is the fact that the "first" Vietoris hyperspace $\mathcal{C}(X)$ of a metrizable consonant space $X$ is hereditarily Baire (i.e., each closed subset of it has the Baire property). This result was achieved in [5] by using measure theoretical arguments that every such hyperspace is a Prohorov space. In Section 4, we relate this property to compact sections of l.s.c. mappings with values in hyperspaces, see Proposition 4.1. However, such properties remain unclear for Cantor set-selectors, see Questions 3 and 4. We conclude the paper with a remark showing that if, in Theorem 1.1, " $Z$ is compact" is replaced by " $Z$ is metrizable" in a suitable way, then the resulting property implies Čech-completeness of a metrizable $X$, Corollary 5.2; in fact, this is derived as a consequence of a known result for a similar modification of the Cantor set-selector property, [23, Theorem 7.1]. 


\section{Proof of Theorem 1.2}

Let $X$ be a metrizable consonant space, $Z$ be a compact metric space, and let $\varphi: Z \rightarrow \mathcal{F}(X)$ be an l.s.c. mapping. Also, let

$$
Y=\operatorname{Graph} \varphi=\{(z, x) \in Z \times X: x \in \varphi(z)\}
$$

be the graph of $\varphi$. Since $\varphi$ is l.s.c. and closed-valued, $Y$ is a $G_{\delta}$-subset of $Z \times X$, see, for instance, [21, Lemma 11.3], also [14, Propositions 6.8 and 6.9]. According to [12, Theorem 7.1], $Z \times X$ also is a consonant space. Since $Z \times X$ is metrizable, by [5, Theorem 6], each $G_{\delta}$-subset of $Z \times X$ is a consonant space, hence so is $Y$. Then, define a mapping $\Phi: Z \rightarrow \mathcal{F}(Y)$ by $\Phi(z)=\{z\} \times \varphi(z), z \in Z$. Since $\varphi$ is l.s.c., so is $\Phi$, and by Theorem 1.1, $\Phi$ has a compact section $K \subset Y$. This $K$ defines a mapping $\theta$ from $Z$ to the subsets of $X$ which has $K$ as its graph, i.e. Graph $\theta=K$. Then, $\theta$ is nonempty-valued because $\varnothing \neq K \cap \Phi(z)=K \cap(\{z\} \times \varphi(z)), z \in Z$, and is also a multi-selection for $\varphi$ because Graph $\theta \subset$ Graph $\varphi$. Finally, $\theta$ is usco because the projection $p_{Z}$ : Graph $\theta \rightarrow Z$ is perfect, see for instance [3, Theorem 2.6], also [8, Proposition 1.1]. The proof of Theorem 1.2 is completed.

Some interesting consequences follow from Theorem 1.2. A classical theorem of Suslin states that every analytic subset of a Polish space is either countable or contains a copy of the Cantor set. For non-separable metrizable spaces, the result has been generalised by Stone [30] (for absolutely Borel spaces), El'kin [13] (for absolutely analytic spaces) and Koumoullis [18] (for Prohorov spaces). According to [16, Theorem 2], every metrizable Cantor set-selector is either scattered or contains a copy of the Cantor set. Consequently, by Theorem 1.2, the same is true for metrizable consonant spaces which allows to get the following results for such spaces.

A space $X$ is dissonant if it is not consonant. It was shown in [25, Examples 9.1 and 9.3] that there are metrizable dissonant spaces; in the same paper (remark added in the proof) there was announced a result by Alleche and Calbrix that there exists a hereditarily Baire separable metrizable dissonant space. The example provided by them is a Bernstein set [1, Corollary 3.6]. This is now an immediate consequence of Theorem 1.2.

\section{Corollary 2.1.}

There exists a separable metrizable space $X$ each closed subset of which is a Baire space, but $X$ is not a consonant space. In fact, every Bernstein space is of this kind.

Proof. A subset $X$ of an uncountable Polish space $Y$ is said to be a Bernstein set if neither $X$ nor $Y \backslash X$ contains a copy of the Cantor set. Each uncountable Polish space contains a Bernstein set [19, p. 514]. According to [16, Theorem 2], every metrizable Cantor set-selector without isolated points contains a copy of the Cantor set. Thus, there is a separable metrizable space $X$ each closed subset of which has the Baire property but $X$ is not consonant. Indeed, by Theorem 1.2, every Bernstein space is of this kind.

Here is another consequence generalising the main result of $[9$, Theorem 1], also simplifying significantly the proof of this fact.

\section{Corollary 2.2.}

Every metrizable consonant space without isolated points contains a copy of the Cantor set. In particular, every metrizable space without isolated points, such that every compact subset of it is scattered, is dissonant.

It follows immediately from Theorem 1.2 and [16, Theorem 2].

\section{Consonance of hyperspaces}

The following simple observation was done in [6], it will be found useful in our next considerations. 
Proposition 3.1.

A regular space $X$ is consonant if and only if for every compact space $Z$, every l.s.c. mapping $\varphi: Z \rightarrow \mathcal{F}(X)$ has a compact section $K \subset X$.

In what follows, we will examine hyperspaces of consonant spaces. Our considerations will involve $\mathcal{C}(X)$ endowed with the Vietoris topology $\tau_{V}$. Recall that $\tau_{V}$ is generated by all collections of the form

$$
\langle\mathcal{V}\rangle=\{S \in \mathcal{C}(X): S \subset \bigcup \mathcal{V} \text { and } S \cap V \neq \varnothing, \text { whenever } V \in \mathcal{V}\}
$$

where $\mathcal{V}$ runs over finite families of open subsets of $X$. For convenience, for an open subset $V \subset X$, we write $\langle V\rangle$ rather than $\langle\{V\}\rangle$. Throughout this paper, $\mathcal{C}(X)$ will always be endowed with the Vietoris topology when it comes to consider it as a topological space.

Let us mention that, for a metric space $(X, d)$, the Vietoris topology on $\mathrm{C}(X)$ is metrizable by the Hausdorff metric

$$
H(d)(S, T)=\inf \left\{\varepsilon>0: S \subset B_{\varepsilon}^{d}(T), T \subset B_{\varepsilon}^{d}(S)\right\}, \quad S, T \in \mathcal{C}(X)
$$

Here, $B_{\varepsilon}^{d}(A)=\{x \in X: d(x, A)<\varepsilon\}$.

Theorem 3.2.

A metrizable space $X$ is consonant if and only if its Vietoris hyperspace $\mathcal{C}(X)$ is consonant.

Proof. For a metrizable $X$, the Vietoris hyperspace $\mathcal{C}(X)$ is also metrizable as mentioned above. If $\mathcal{C}(X)$ is consonant, then each closed subset of it also is consonant [12, Proposition 4.2], see also Proposition 3.1. Since $x \mapsto\{x\}, x \in X$, is an embedding of $X$ in $\mathcal{C}(X)$ as a closed subset, $X$ itself is a consonant space. Suppose now that $X$ is consonant, and take an l.s.c. mapping $\varphi: Z \rightarrow \mathcal{F}(\mathcal{C}(X))$ for some compact space $Z$. We follow the idea of [5, Proposition 4]. Namely, let $\mathcal{H}$ be the family of all open subsets $V \subset X$ such that $\langle V\rangle$ is a section for $\varphi$. Since $Z$ is compact and $\varphi$ is l.s.c., this is a nonempty compact family (i.e., a Scott open set). Then, $\mathcal{H}$ is compactly generated. Take $U \in \mathcal{H}$ and let $K \subset U$ be a compact subset of $X$ such that $V \in \mathcal{H}$ for every open $V \subset X$, with $K \subset V$. If $z \in Z$ and $K \cap \overline{U \varphi(z)}=\varnothing$, then $V=X \backslash \overline{\bigcup \varphi(z)}$ is an open set such that $K \subset V$, hence $V \in \mathcal{H}$, but $\langle V\rangle$ is not a section for $\varphi$, and so $V \notin \mathcal{H}$. Thus, we must have that $K \cap \overline{U \varphi(z)} \neq \varnothing$. So, $K$ is a nonempty compact subset of $X$, and we may consider the nonempty compact subset $\mathcal{C}(K)$ of $\mathcal{C}(X)$. Suppose that $\mathcal{C}(K) \cap \varphi(z)=\varnothing$ and take a metric $d$ on $X$ compatible with the topology of $X$. Since $\mathcal{C}(K)$ is a compact subset of $\mathcal{C}(X)$ disjoint from the closed subset $\varphi(z)$, there exists $\varepsilon>0$ such that $B_{\varepsilon}^{H(d)}(\mathcal{C}(K)) \cap \varphi(z)=\varnothing$. According to the definition of $H(d)$ and the fact that $\mathcal{C}(K)$ consists of all nonempty compact subsets of $K$, we now have $K \in\left\langle B_{\varepsilon}^{d}(K)\right\rangle \subset B_{\varepsilon}^{H(d)}(\mathcal{C}(K))$ and, in particular, that $\left\langle B_{\varepsilon}^{d}(K)\right\rangle$ is not a section for $\varphi$, while by the property of $K$ we also have that $B_{\varepsilon}^{d}(K) \in \mathcal{H}$. A contradiction. Thus, $\mathcal{C}(K)$ is a section for $\varphi$ and, by Proposition 3.1, the proof is completed.

Motivated by Theorem 3.2, to every metrizable space $X$ we may associate the sequence of hyperspaces $\mathcal{C}^{(n)}(X), n<\omega$, defined by

$$
\mathcal{C}^{(0)}(X)=X \quad \text { and } \quad \mathcal{C}^{(n+1)}(X)=\mathcal{C}\left(\mathcal{C}^{(n)}(X)\right) .
$$

It now follows from Theorem 3.2 that consonance is stable with respect to these hyperspaces.

\section{Corollary 3.3.}

If a metrizable space $X$ is consonant, then each $\mathrm{C}^{(n)}(X), n<\omega$, is also consonant.

Combining Theorems 1.2 and 3.2, we also have the following interesting consequence which reveals more about the relationship between consonant spaces and Cantor set-selectors.

\section{Corollary 3.4.}

If a metrizable space $X$ is consonant, then each $\mathrm{C}^{(n)}(X), n<\omega$, is a Cantor set-selector. 
Motivated by Corollary 3.4, we have the following natural question.

\section{Question 2.}

Does there exist a metrizable Cantor set-selector $X$ such that $\mathcal{C}(X)$ is not a Cantor set-selector?

Of course, if the answer to Question 2 is "yes", then such a space must be dissonant.

\section{Prohorov spaces}

Let $\mathcal{B}(X)$ be the smallest $\sigma$-algebra that contains all closed subsets of a space $X$, i.e. the Borel $\sigma$-algebra associated to $X$; the elements of $\mathcal{B}(X)$ are often called Borel subsets of $X$. A countably additive function $\mu: \mathcal{B}(X) \rightarrow[0,+\infty]$ is a Radon measure if

$$
\mu(B)=\sup \{\mu(K): K \subset B, K \text { is compact }\}, \quad B \in \mathcal{B}(X) .
$$

A Radon probability measure is a Radon measure $\mu$ with $\mu(X)=1$, and we use $\mathcal{P}(X)$ to denote the set of all such measures. Every $\mu \in \mathcal{P}(X)$ uniquely defines a positive linear functional $\mu(g)=\int g d \mu$, where $g$ runs over bounded continuous functions on $X$. As a topological space, we consider $\mathcal{P}(X)$ endowed with the weakest topology with respect to which all these functionals are continuous. Thus, a net $\left\{\mu_{\alpha}\right\} \subset \mathcal{P}(X)$ converges to $\mu \in \mathcal{P}(X)$ if and only if $\left\{\mu_{\alpha}(g)\right\}$ converges to $\mu(g)$ for every bounded continuous function $g: X \rightarrow \mathbb{R}$. In particular, for every closed $F \subset X$ and $\varepsilon>0$, the set $\{\mu \in \mathcal{P}(X): \mu(F)<\varepsilon\}$ is open in $\mathcal{P}(X)$.

The famous Prohorov theorem [27] states that if $X$ is a Polish space, then for every compact $Z \subset \mathcal{P}(X)$ and every $\varepsilon>0$ there exists a compact $K \subset X$, with $\mu(X \backslash K)<\varepsilon$ for all $\mu \in Z$. Spaces having this property, called Prohorov spaces, are widely investigated in the literature. It is well known that Čech-complete spaces are Prohorov [26], and more generally all sieve-complete spaces, see [17].

Prohorov spaces are defined by compact sets and behave in a similar way as consonant spaces. For instance, it was proved in [26, Theorem 6] that a co-analytic separable metrizable space is Prohorov if and only if it is Čech-complete; also that every $G_{\delta}$-subset of a Prohorov space is a Prohorov space [26, Theorem 1].

Prohorov spaces have been the main interface between consonant spaces and hereditarily Baire spaces. Let us mention Debs' result [10, Théorème 5.1] that every Prohorov space having a dense first countable subspace is a Baire space. On the other hand, it was proved by Bouziad [4, Theorem 2.2] that every completely regular consonant space is Prohorov. It was further proved by Bouziad [5, Proposition 4] that the hyperspace $\mathcal{C}(X)$ is a Prohorov space for every completely regular consonant space $X$. Combining these results, Bouziad derived in [5, Proposition 5] that $\mathrm{e}(X)$ is hereditarily Baire for every metrizable consonant space $X$. The following observation follows the idea of the proof of [17, Theorem 3.1], and offers some explanation for this relationship.

\section{Proposition 4.1.}

Let $X$ be a metrizable space such that every l.s.c. mapping $\varphi: \mathfrak{C} \rightarrow \mathcal{F}(\mathcal{C}(X))$ has a compact section $\mathcal{K} \subset \mathcal{C}(X)$. Then, $X$ is a Prohorov space.

Proof. Given $\varepsilon>0$, define a mapping $\Psi$ from $\mathcal{P}(X)$ to the subsets of $\mathcal{C}(X)$ by

$$
\Psi(\mu)=\{K \in \mathcal{C}(X): \mu(X \backslash K)<\varepsilon\}, \quad \mu \in \mathcal{P}(X),
$$

and let $\Phi(\mu)$ be the $\tau_{V}$-closure of $\Psi(\mu)$ in $\mathcal{C}(X)$, for each $\mu \in \mathcal{P}(X)$. By [17, Proposition 2.1] and [20, Proposition 2.3], $\Phi: \mathcal{P}(X) \rightarrow \mathcal{F}(\mathcal{C}(X))$ and it is l.s.c. Take a compact subset $Z \subset \mathcal{P}(X)$. According to [2, Theorem 2.27], $\mathcal{P}(X)$ is metrizable, hence $Z$ is a compact metrizable space (in particular, a continuous image of $\mathfrak{C}$ ), and, by hypothesis, the l.s.c. mapping $\varphi=\Phi\lceil Z$ has a compact section $\mathcal{K} \subset \mathcal{C}(X)$. Then, $K=\bigcup \mathcal{K}$ is a compact subset of $X$, see for instance [17, Proposition 2.3]. Finally, take $\mu \in Z$ and $A \in \mathcal{K} \cap \varphi(\mu) \subset \Phi(\mu)$. By [17, Proposition 2.2], $\mu(X \backslash K) \leq \mu(X \backslash A) \leq \varepsilon$, i.e. $X$ is a Prohorov space. 
According to Debs' result [10, Théorème 5.1] and Corollary 3.3, we have

\section{Corollary 4.2.}

If $X$ is a metrizable consonant space, then each $\mathrm{C}^{(n)}(X), n<\omega$, is a Prohorov space. In particular, each $\mathrm{C}^{(n)}(X), n<\omega$, is hereditarily Baire.

The property that each $G_{\delta}$-subset of a metrizable consonant space $X$ is also consonant [5, Theorem 6], is essentially based on the fact that $\mathcal{C}(X)$ is hereditarily Baire. In turn, this property was the most essential one to show that every metrizable consonant space is a Cantor set-selector. It seems, the situation with hyperspaces of metrizable Cantor set-selectors is unclear; for instance, the following question is still open.

Question 3 ([16]).

Is every $G_{\delta}$-subset of a metrizable Cantor set-selector also a Cantor set-selector?

The above question can be resolved affirmatively following the idea of [5, Theorem 6] provided that the Vietoris hyperspace $\mathfrak{C}(\mathfrak{C} \times X)$ is hereditarily Baire for a metrizable $\mathfrak{C}$-selector $X$.

According to [16, Theorem 1] (which has a very simple direct proof), every metrizable Cantor set-selector is hereditarily Baire. However, Cantor set-selectors were defined by the existence of u.s.c. multi-selections which are not necessarily compact-valued. In this regard, let us agree that a space $X$ is a usco $\mathfrak{C}$-selector if every l.s.c. mapping $\varphi: \mathfrak{C} \rightarrow \mathcal{F}(X)$ has a usco multi-selection $\theta: \mathfrak{C} \rightarrow \mathcal{C}(X)$. We now have also the following natural question.

\section{Question 4.}

Is every metrizable usco $\mathfrak{C}$-selector a Prohorov space?

\section{Consonance and complete metrizability}

As it was mentioned in Introduction, a metrizable space $X$ is Čech-complete iff for every paracompact space $Z$, every l.s.c. mapping $\varphi: Z \rightarrow \mathcal{F}(X)$ has a usco multi-selection $\theta: Z \rightarrow \mathcal{C}(X)$. In fact, a bit stronger result was proved in [23] that it suffices to consider this property only for metrizable spaces $Z$. To this end, recall that a mapping $\varphi: Z \rightarrow \mathcal{F}(X)$ from a space $Z$ to the subsets of a metric space $(X, d)$ is $d$-u.s.c. (respectively, $d$-l.s.c.) if for every $\varepsilon>0$, every $z_{0} \in Z$ has a neighbourhood $U$ such that $\varphi(z) \subset B_{\varepsilon}^{d}\left(\varphi\left(z_{0}\right)\right)$ (respectively, $\varphi\left(z_{0}\right) \subset B_{\varepsilon}^{d}(\varphi(z))$ ) for every $z \in U$. A mapping $\varphi: Z \rightarrow \mathcal{F}(X)$ is called $d$-continuous (sometimes, also, Hausdorff continuous) if it is both $d$-l.s.c. and $d$-u.s.c. Let us remark that every $d$-l.s.c. mapping is l.s.c. and every u.s.c. is $d$-u.s.c., but neither of these is invertible.

\section{Theorem 5.1 ([23, Theorem 7.1]).}

Let $(X, d)$ be a metric space such that for every metrizable $Z$, every $d$-continuous mapping $\varphi: Z \rightarrow \mathcal{F}(X)$ has a usco multi-selection $\psi: Z \rightarrow \mathcal{C}(X)$. Then, $X$ is Čech-complete.

Theorem 5.1 implies the following consequence which sheds also some light on [15, Question 1].

\section{Corollary 5.2.}

Let $X$ be a metrizable space such that for every metrizable $Z$, every l.s.c. mapping $\varphi: Z \rightarrow \mathcal{F}(X)$ has a usco section $\theta: Z \rightarrow \mathcal{C}(X)$. Then, $X$ is Čech-complete.

Proof. Let $Z$ be metrizable, $d$ be a compatible metric on $X, \varphi: Z \rightarrow \mathcal{F}(X)$ be $d$-continuous and $\theta: Z \rightarrow \mathcal{C}(X)$ be a usco section for $\varphi$. Define another mapping $\psi: Z \rightarrow \mathcal{C}(X)$ by $\psi(z)=\theta(z) \cap \varphi(z), z \in Z$. Then, $\psi$ is a multi-selection for $\varphi$ and, by Theorem 5.1, it suffices to show that $\psi$ is usco. Since $\varphi$ is $d$-continuous, its graph Graph $\varphi$ is closed 
in $Z \times X$. Since $\theta$ is usco, its graph Graph $\theta$ also is closed in $Z \times X$ and $\pi_{Z}\left\lceil\right.$ Graph $\theta$ is perfect, where $\pi_{Z}: Z \times X \rightarrow X$ is the projection. According to the definition of $\psi$, Graph $\psi=\operatorname{Graph} \theta \cap \operatorname{Graph} \varphi$, hence Graph $\psi$ is closed in Graph $\theta$, and $\pi_{Z}\lceil$ Graph $\psi$ is perfect as well. Thus, $\psi$ is usco.

\section{Acknowledgements}

The author would like to express his best gratitude to Professor Vesko Valov for several valuable comments.

\section{References}

[1] Alleche B., Calbrix J., On the coincidence of the upper Kuratowski topology with the cocompact topology, Topology Appl., 1999, 93(3), 207-218

[2] Banakh T.O., Topology of spaces of probability measures. I. The functors $P_{\tau}$ and $\widetilde{P}$, Mat. Stud., 1995, 5, 65-87 (in Russian)

[3] Borges C.J.R., A study of multivalued functions, Pacific J. Math., 1967, 23, 451-461

[4] Bouziad A., Borel measures in consonant spaces, Topology Appl., 1996, 70(2-3), 125-132

[5] Bouziad A., A note on consonance of $G_{\delta}$ subsets, Topology Appl., 1998, 87(1), 53-61

[6] Bouziad A., Consonance and topological completeness in analytic spaces, Proc. Amer. Math. Soc., 1999, 127(12), 3733-3737

[7] Bouziad A., Filters, consonance and hereditary Baireness, Topology Appl., 2000, 104(1-3), 27-38

[8] Choban M.M., Many-valued mappings and Borel sets. I, Trans. Moscow Math. Soc., 1970, 22, 258-280

[9] Costantini C., Watson S., On the dissonance of some metrizable spaces, Topology Appl., 1998, 84(1-3), 259-268

[10] Debs G., Espaces héréditairement de Baire, Fund. Math., 1988, 129(3), 199-206

[11] Dolecki S., Greco G.H., Lechicki A., Sur la topologie de la convergence supérieure de Kuratowski, C. R. Acad. Sci. Paris, 1991, 312(12), 923-926

[12] Dolecki S., Greco G.H., Lechicki A., When do the upper Kuratowski topology (homeomorphically, Scott topology) and the co-compact topology coincide?, Trans. Amer. Math. Soc., 1995, 347(8), 2869-2884

[13] El'kin A.G., A-sets in complete metric spaces, Dokl. Akad. Nauk SSSR, 1967, 175, 517-520

[14] Gutev V., Selections and approximations in finite-dimensional spaces, Topology Appl., 2005, 146-147, 353-383

[15] Gutev V., Completeness, sections and selections, Set-Valued Anal., 2007, 15(3), 275-295

[16] Gutev V., Nedev S., Pelant J., Valov V., Cantor set selectors, Topology Appl., 1992, 44(1-3), 163-166

[17] Gutev V., Valov V., Sections, selections and Prohorov's theorem, J. Math. Anal. Appl., 2009, 360(2), 377-379

[18] Koumoullis G., Cantor sets in Prohorov spaces, Fund. Math., 1984, 124(2), 155-161

[19] Kuratowski K., Topology. I, Academic Press, New York-London; PWN, Warsaw, 1966

[20] Michael E., Continuous selections. I, Ann. of Math., 1956, 63, 361-382

[21] Michael E., Continuous selections. II, Ann. of Math., 1956, 64, 562-580

[22] Michael E., A theorem on semi-continuous set-valued functions, Duke Math. J., 1959, 26, 647-651

[23] van Mill J., Pelant J., Pol R., Selections that characterize topological completeness, Fund. Math., 1996, 149(2), 127-141

[24] Nedev S.J., Valov V.M., On metrizability of selectors, C. R. Acad. Bulgare Sci., 1983, 36(11), 1363-1366

[25] Nogura T., Shakhmatov D., When does the Fell topology on a hyperspace of closed sets coincide with the meet of the upper Kuratowski and the lower Vietoris topologies?, Topology Appl., 1996, 70(2-3), 213-243

[26] Preiss D., Metric spaces in which Prohorov's theorem is not valid, Z. Wahrscheinlichkeitstheorie und Verw. Gebiete, 1973, 27, 109-116

[27] Prokhorov Yu.V., Convergence of random processes and limit theorems in probability theory, Theory Probab. Appl., 1956, 1(2), 157-214

[28] Przymusiński T., Collectionwise normality and absolute retracts, Fund. Math., 1978, 98(1), 61-73 
[29] Scott D., Continuous lattices, In: Toposes, Algebraic Geometry and Logic, Halifax, January 16-19, 1971, Lecture Notes in Math., 274, Springer, Berlin, 1972, 97-136

[30] Stone A.H., On $\sigma$-discreteness and Borel isomorphism, Amer. J. Math., 1963, 85, 655-666 